\title{
A Novel Approach of Cluster based Routing in Mobile Ad hoc Networks
}

\author{
Jagadish Gurrala \\ Assistant Professor \\ Anil Neerukonda Institute of \\ Technology and Sciences \\ Visakhapatnam
}

\author{
Paila Usha \\ Assistant Professor \\ ASK Engineering College \\ Anakapalli,Vsp
}

\author{
Soumya Ranjan Mishra \\ Assistant Professor \\ Anil Neerukonda Institute of \\ Technology and Sciences \\ Visakhapatnam
}

\begin{abstract}
The research on the various issues in Mobile ad hoc networks are getting popularity because of its challenging nature and all time connectivity to communicate . MANET is a random deployable network where devices mobile with dynamic topology. In the network topology, each device is termed as a node and the virtual connectivity among each node is termed as the link. Nodes in the network are dynamically organized into virtual partitions called cluster. Network simulators provide the platform to analyze the working of computer networks along with the typical devices, traffic and other entities using ns2. In this paper we analyze and provide ways of how nodes are made for routing after clustering among nodes during simulation.
\end{abstract}

\section{General Terms}

Clustering Scheme, Mobile adhoc Network.

\section{Keywords}

Transceiver, Packet Switching,

\section{INTRODUCTION}

Nodes/transceiver are distinguished by limited resources such as power and mobility. Due to the limited transmission range of the nodes, multi hops may be needed for a node to send data to any other node in the network (1]. If a transceiver wants to communicate with another node that is outside its transmission range an intermediate node acts as a router to relay or forward packets from the source to destination. The major reason for this is because the earlier networks were very simple interconnected structure of transceiver and homogeneous environments; but, now high end and large scale internetworking has become popular with the latest advancements in the networks and telecommunication technology. The routing concept basically involves, two activities: firstly, determining optimal routing paths and secondly, transferring the information groups (called packets) through an internetwork. The later concept is called as packet switching which is straight forward, and the path determination could be very complex. For this a routing protocol is required. Nodes in Ad-hoc networks are free to move over a certain area. Because of this movement, the network topology changes. The design of the protocol is essential due to the dynamism of the network. MANETs are characterized by the absence of any centralized co-ordination or fixed infrastructure, which makes any node in the network act as a potential router. They have dynamic, random and quickly changing topology. Within a cell, a base station can reach all mobile nodes without routing via broadcast in common wireless networks. But in different cells, since no infrastructure exists and due to node mobility frequent link failure occurs. Hence, it is a great challenge to design an effective and adaptive routing protocol considering limited bandwidth and power.

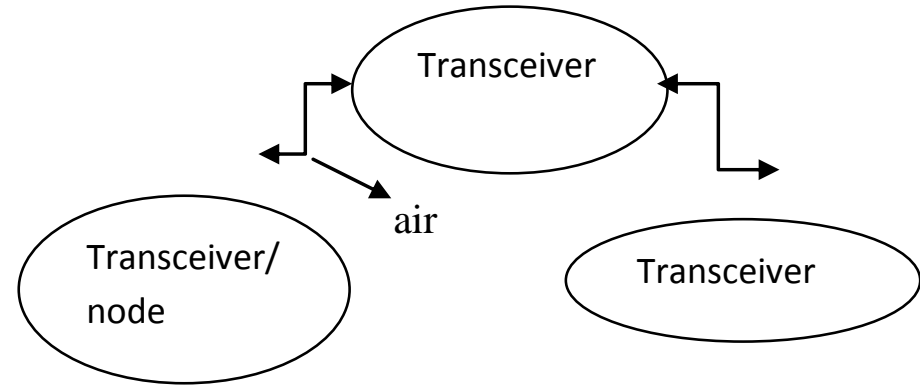

Fig 1.1 Interconnection of transceiver through air interface in MANET

The rest of this paper is organized as follows. In section 2 discuss literature review on clustering concept were presented. In section 3 focuses on related work and simulation setup and running a scenarios were discussed in section 4 . In section 5 the results of the performance evaluation are discussed. Conclusion \& Future work is given in section 6 .

\section{LITERATURE REVIEW}

\subsection{Problem Statement}

Cluster based routing problems are exist in MANET's and there are two signification problems[2] faced in MANET. First reason is MANET is affected with odd cluster formation due to mobility of nodes and frequent changes in topology, and second reason is the mobile nodes has limited network life time with more power consumption in data transfer.Our project contains three modules such as Node placement, Cluster formation and Routing in Intra cluster communication[3]. The simulation results carried in terms of graphs using XGraph utilities in NS2, which shows the graphs shows packet delivery ratio vs nodes and throughput vs nodes.

\section{CLUSTERING CONCEPT}

Clustering is one approach to reduce traffic during the routing process, and several authors proposed cluster based routing protocols. All the nodes are grouped into clusters and each cluster[4] has one cluster head in addition to many gateways. The cluster head is responsible for its cluster member, whose rebroadcast can cover all nodes in that cluster. One of the leading protocols in MANET is Cluster Based Routing Protocol(CBRP)[12], is an demand routing protocol, where nodes are divided into clusters. Initially each node in the network has undecided state.Node starts timer and broadcasts HELLO packet. If it receives a Hello reply from any cluster head then sets it state as cluster member else it makes itself as cluster head but only when it has bidirectional links with one or more neighbor nodes. Otherwise it repeats the procedure with sending HELLO packets. Clustering[5] is the process that divides the network into interconnected substructures, called clusters. Each cluster has a cluster head and act as a 
coordinator within the substructure. Each cluster head, in other terms acts as a temporary base station with in its zone or cluster and communicates with its peers. Clustering algorithm[6] used in CBRP is a variation of simple "lowest ID" clustering algorithm in which the node with a lowest ID among its neighbors is elected as the Cluster head. Each Node maintains neighbor table and cluster adjacency table[7].

\subsection{Architecture:}

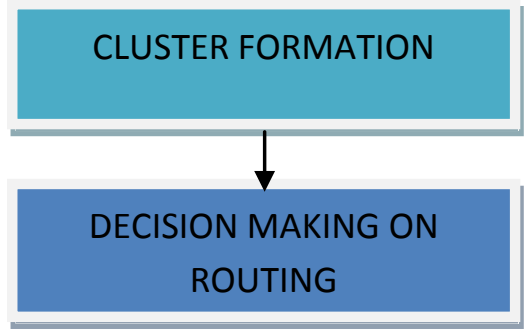

\subsection{Routing Algorithm}

$\mathrm{CH}=$ cluster head

Initially source node(A) sends the packet to the to its $\mathrm{CH}[12]$.

Step1: if the destination node is with in the cluster it ends directly to the destination.

Step2: Else sends the packet to the gateway. Then it selects the shortest path for transferring.

Step3: packets are send through the intermediate cluster gateways

Step4: after that the destination gateway receives the packets.

Step5: finally packets forward to the destination.

\section{THROUGHPUT:}

Throughput is the ratio of number of packets received to time in seconds

\section{Throughput $=\underline{\text { No. of packets received }}$ Simulation time (msec)}

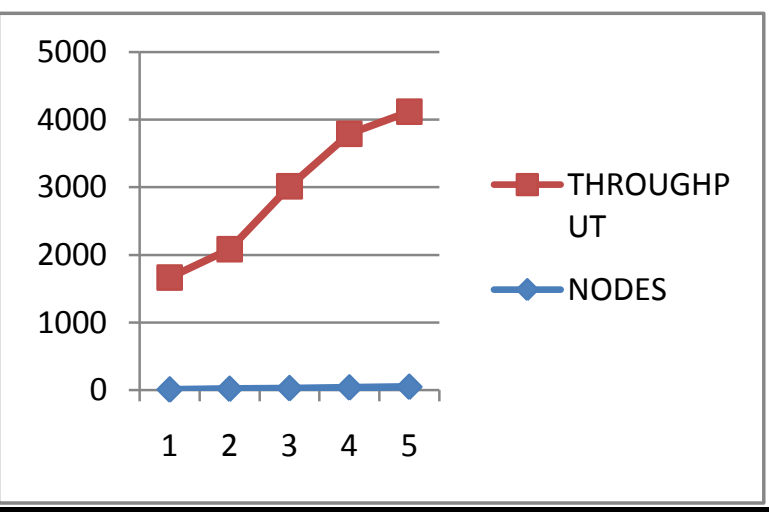

Table 1. Extract values Nodes Vs DSDV from trace file

\begin{tabular}{|l|l|l|l|l|l|}
\hline Nodes & 10 & 20 & 30 & 40 & 50 \\
\hline DSDV & $\begin{array}{l}1654.99 \\
8\end{array}$ & 2061.667 & 2984.3 & 3748.77 & 4065.86 \\
& & & 38 & 2 & 4 \\
\hline
\end{tabular}

\subsection{Packet Delivery Fraction (PDF):}

This is the ratio of total number of packets successfully received by the destination nodes to the number of packets sent by the source nodes throughout the simulation[9].

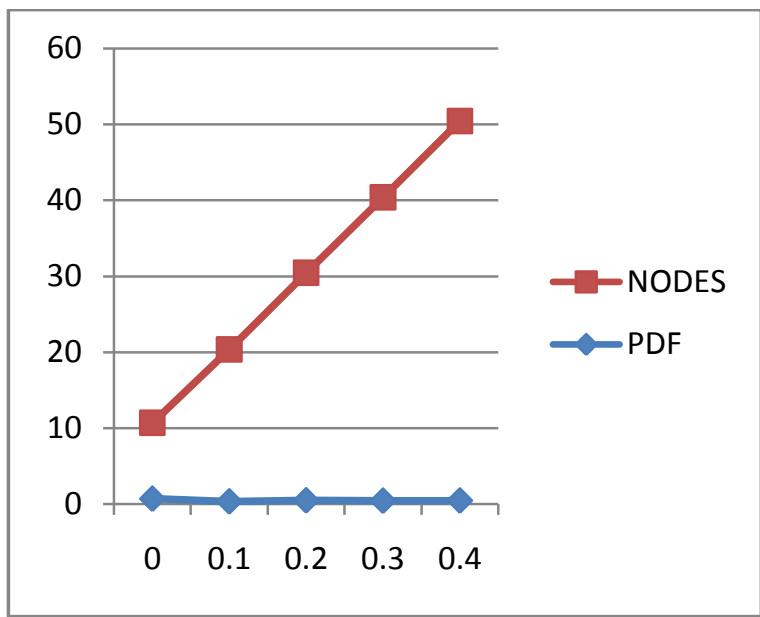

\begin{tabular}{|c|c|c|c|c|c|}
\hline Nodes & 10 & 20 & 30 & 40 & 50 \\
\hline DSDV & 0.6709 & 0.3217 & 0.4568 & 0.4067 & 0.4363 \\
\hline
\end{tabular}

5. SCREENSHOTS

\subsection{Visualization of a Wireless Scenario with 50 Nodes using NAM}

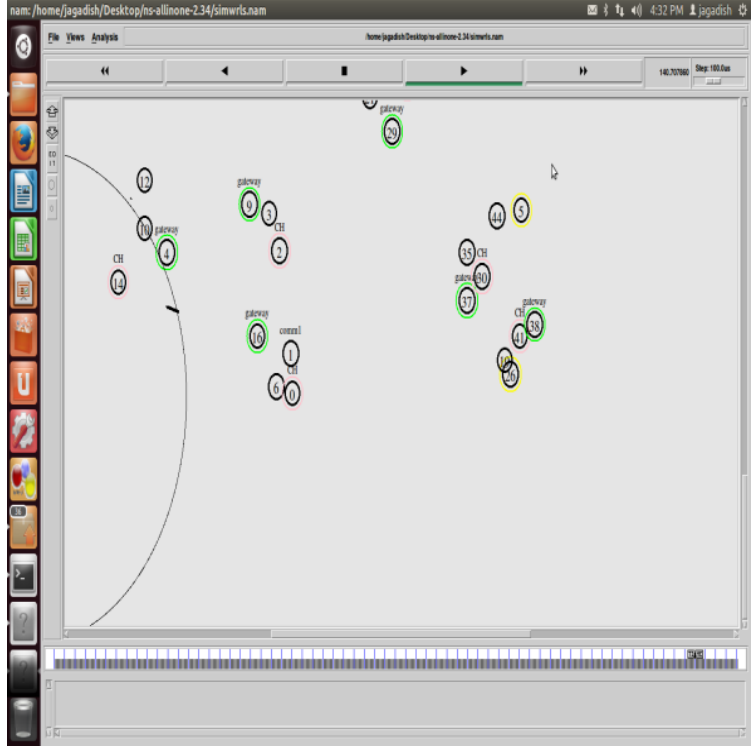




\subsection{Output of Trace File :}

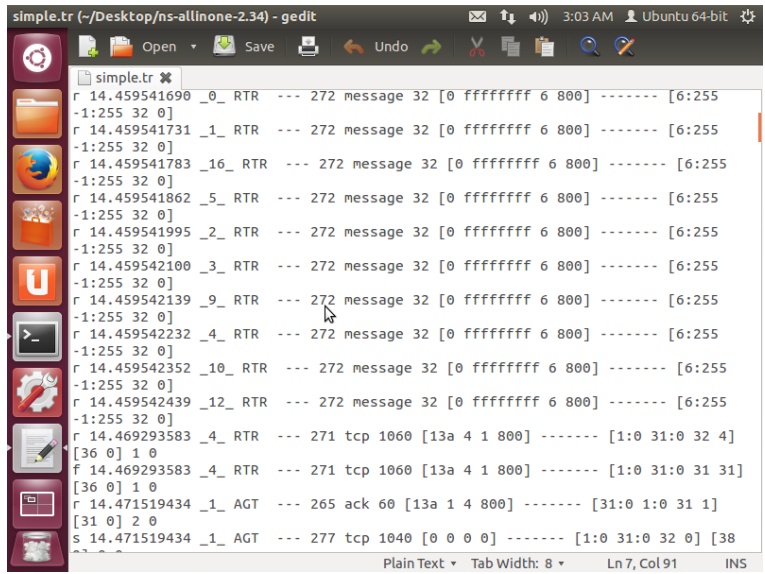

\subsection{Output for Packet Transmission:}

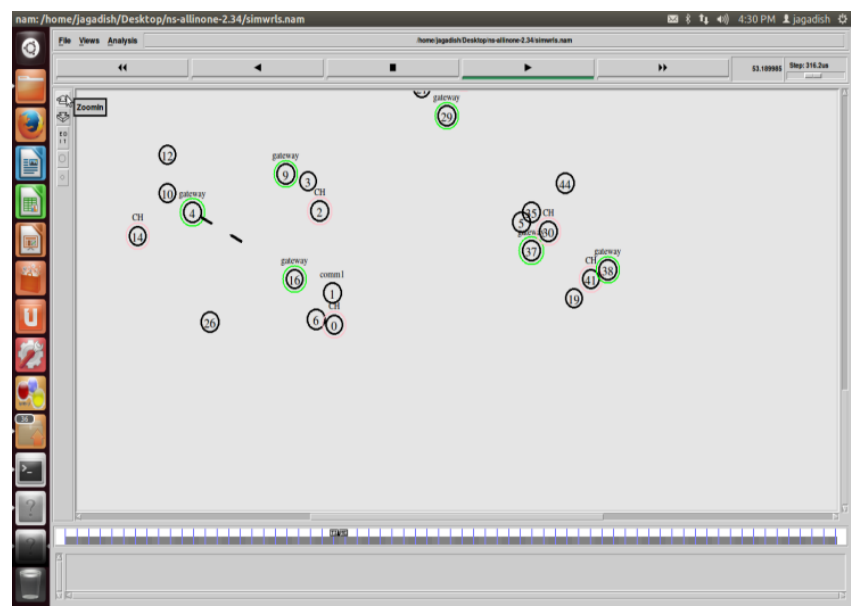

\subsection{Graph for Packet Delivery Ratio:}

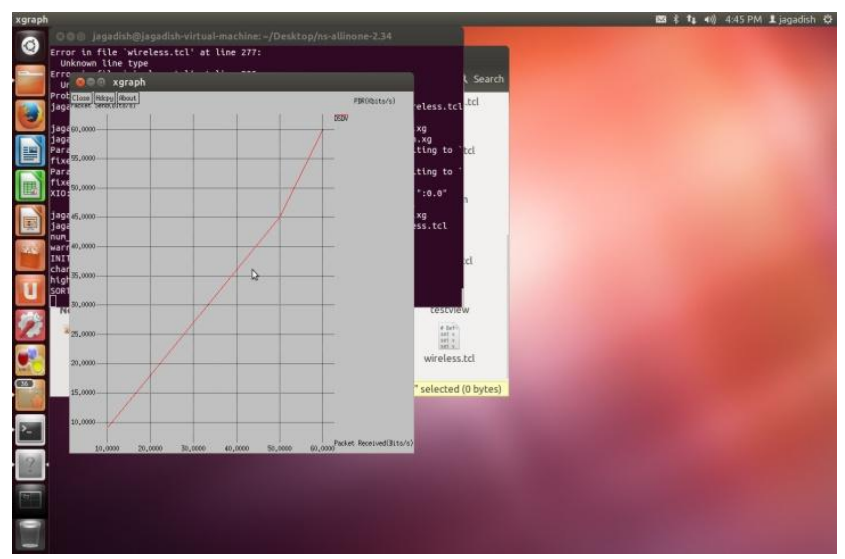

\section{INSTALLING UBUNTU:}

Initially install VM ware Workstation in our computer having windows Operating system . Now open VM ware Workstation and select new virtual Machine[10] option select typical radio button and click next button . Now Click the radio button $\mathrm{i}$ will install the operating system later and click next Button .Select Linux and version as ubuntu 64-bit click next button and write .The virtual machine name[11] as ubuntu and select next button, enter maximum disk size as $20 \mathrm{gb}$ and click the radio button named as store virtual disk as a single file and next button. Now ready to create virtual machine will be displayed click finish button to create virtual machine.

Now double click on the extracted file of ubuntu operating system select Extract button and go to User Name->My Documents->Virtual Machines and Click ubuntu folder and click ok button then extraction will be done in that ubuntu folder. By this way ubuntu has been installed.

Open VMware and click ubuntu, this operating system will be opened and prompt to enter user name and password ,after validation ubuntu operating system is ready to use.

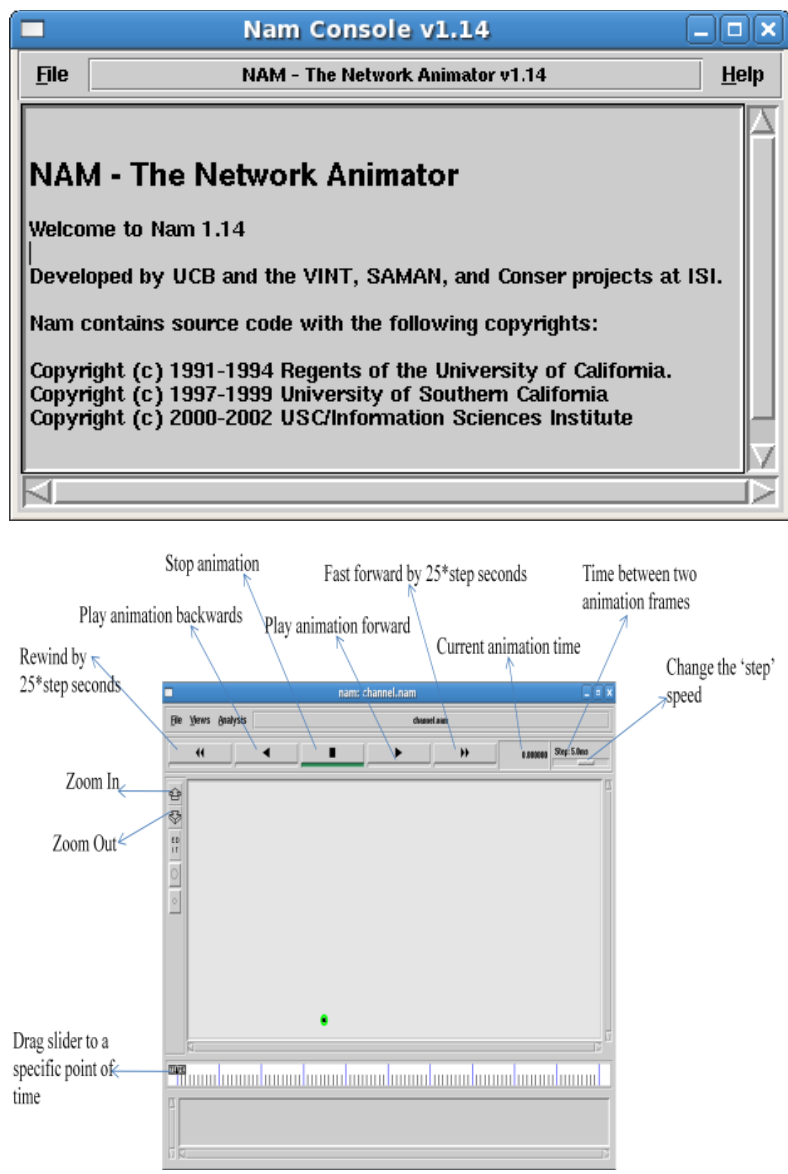

\subsection{Xgraph Utility in NS2:}

Xgraph is a plotting program which can be used to create graphic representations of simulation results. You can create output files in your Tcl scripts, which can be used as data sets for xgraph. Call xgraph to display the results with thecommand "xgraph <data-file>".

The xgraph program[8] draws a graph on an $\mathrm{X}$ display given data read from either data files or from standard input if no files are specified. It can display up to 64 independent data sets using different colors and/or line styles for each set. It annotates the graph with a title, axis labels, grid lines or tick marks and grid labels. 


\subsection{Xgraph of Packets Received and Lost of Dsdv of 50 Nodes:}

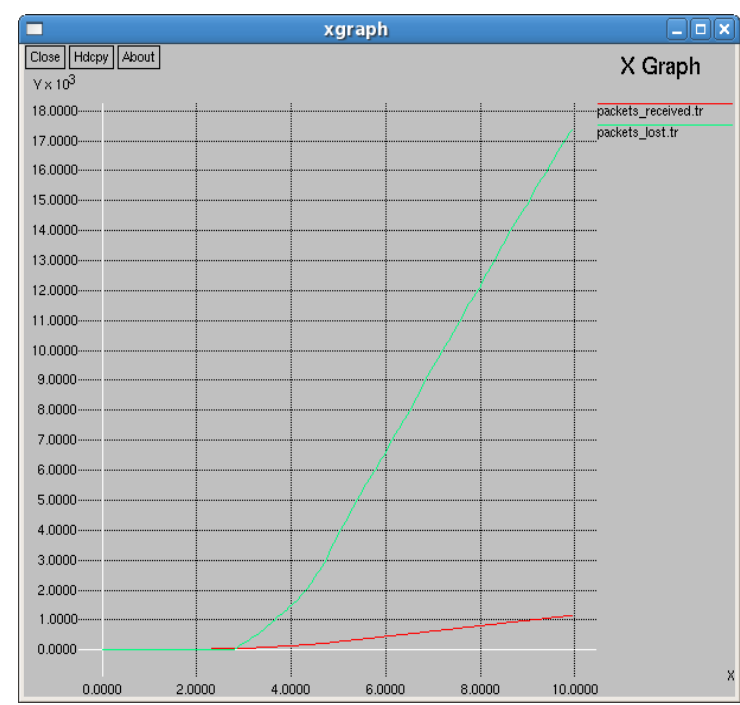

\subsection{Troubleshooting:}

1. Problem: "Some test failed" during validation in installation process of NS2.

Solution: This error occurs when there is compatibility issues with the packages present in the Operating System. This can be solved by updating the packages[13] or replacing the Operating System which is best compatible with NS2.In our paper is compatible with NS-2.34 with Fedora 7.

2. Problem: "ns command not found" while executing a TCL script.

Solution: This error occurs for any of the two reasons:

NS2 may not be installed properly. If this is the case, reinstalling the NS2 may solve the issue.

Path for NS is not set or wrongly set. Setting the path correctly solves the issue.

3. Problem: "nam command not found" while executing Network Animator.

Solution: This error occurs for any of the two reasons:

NS2 may not be installed properly. If this is the case, reinstalling the NS2 may solve the issue.

Path for NAM is not set or wrongly set. Setting the path correctly solves the issue.

\section{Problem: "No data to plot" while executing Xgraph.}

Solution: This error occurs when the input file i.e, text file does no consists of the data which is to be plotted. This can be solved by extracting the data into the text file which is to be plotted.

\section{CONCLUSION AND FUTURE WORK:}

A network simulator is a tool that analyzes the working of a computer network. In simulators, the computer network is typically modeled with devices, traffic etc and the performance is analyzed. Typically, users can then customize the simulator to fulfill their specific analysis needs. The proposed simulator is an extension to the earlier work for designing of a distributed clustering algorithm for ad hoc networks. A node that consumes minimum energy in the network is selected as the cluster head. The energy consumption is considered to be the function of the degree of connectivity of a node along with its average transmission power that is consumed in serving its neighbor nodes. In future routing protocols can also be implemented with further modification.

\section{ACKNOWLEDGMENTS}

The authors would like to thank Shri. P.Sanyasi Naidu, Associate Professor, Dept. of CSE, GITAM University, India and Prof. S. C Satapathy, CSE Department, ANITS for their support to carry out experimentation and programming work.

\section{REFERENCES:}

[1] https://sites.google.com/site/rsetnsworkshop/awk-xgraph

[2] http://www.seminarsonly.com/IT/Tool-CommandLanguage.php

[3] S. Basagni, "Distributed clustering for ad hoc networks," Proceedings of International Symposium on Parallel Architecture,Algorithms and Networks, June 1999.

[4] http://byuvraj.blogspot.in/

[5] http://www.powershow.com/view/156316-OTdiN/

[6] http://mohittahiliani.blogspot.in/2010/02/few-more-awkscripts-for-ns2.html.

[7] http://books.google.co.in/books?id=Cf570PJAguIC\&pg= PA501\&dq $=$ new + trace + file + format + of $+n s+2.34 \&$ hl $=$ en $\&$ sa $=$ X\&ei=WtwUZapGMW3rAfB7IGgAg\#v=onepage \&q\&f=false

[8] http://mohittahiliani.blogspot.in/2009/12/awk-script-forns2.htmlTutorial_The_ns2_Network_Simulator_powerp oint_ppt_presentation

[9] http://www.vectorsite.net/tsawk.html

[10] http://hpds.ee.ncku.edu.tw/ smallko/ns2/adhoc_routing.h tml.

[11] K. X. Xu, X. Y. Hong, and M. Gerla, "An Ad Hoc Network with Mobile Backbones," Proc. IEEE ICC'2002, vol. 5, Apr.-May2002, pp. 313.

[12] Safa, H.; Artail, H.; Tabet, D.: A cluster-based trustaware routing protocol for mobile ad-hoc networks. $\mathrm{J}$. Wirel. Netw. 16, 969--984 (2010)

[13] Jane y. yu and peter h. j. chong, A survey of clustering schemes for mobile adhoc networks, IEEE ommunication 2005 , volume 7 , no. 1 . 